\title{
The Comparison of Mental Rotation Performance in Team and Individual Sports of Students
}

\author{
Fatemeh Pasand (Corresponding author) \\ Department of Physical Education, Shiraz University, Iran \\ E-mail: pasand@shirazu.ac.ir \\ Azam Rekabi \\ Department of Physical Education, Shiraz University, Iran \\ E-mail: Maryam.al55@yahoo.com \\ Ali Mohammad Goodarzi \\ Department of clinical Psychology, Shiraz University, Iran \\ E-mail: mgoodarzi@rose.shirazu.ac.ir \\ Majid Chahardahcheric \\ Department of Physical Education, Shiraz University, Iran \\ E-mail: Majid chahardahcheric@yahoo.com
}

Received: 15-12- 2014

doi:10.7575/aiac.ijkss.v.3n.1p.21
Accepted: 26-01- 2015

URL: http://dx.doi.org/10.7575/aiac.ijkss.v.3n.1p.21
Published: 31-01- 2015

\begin{abstract}
As a practical and causal-comparative study, the present study was aimed at comparing the mental rotation performance in team and individual sports among students. The statistical population included all of the female and male athletes $(\mathrm{N}=1500)$ from different districts of Shiraz, Iran who participated in the sport clubs. The participants of this study included 240 students between 12-14 years old (120 girls and 120 boys) who were selected randomly from four sport fields (Volleyball, Basketball, Karate, and Gymnastics). Finally, 30 athletes were selected from each field. The Mentrat Program, a kind of software for the Mental Rotation Test was used as an evaluation tool. Analyses of variance (ANOVA) with repeated measures were conducted to analysis of data. The results indicated that the impact of the rotational angle was significant in both team and individual groups $(p<0.05)$ while there was not any significant difference in the mental rotation performance in the team and individual sports of the male and female participants $(\mathrm{p}>0.05)$. It was also observed that there was a significant difference between the mental rotation scores of the males in the individual groups contrary to the ones in the team groups $(\mathrm{p}<0.05)$. As a whole, it seems that as the rotational angle increases, the ability of the mental rotation in the individual fields of sport (males) will be higher compared to the team groups.
\end{abstract}

Keywords: Mental Rotation, Rotational Angle, Team and Individual Sports, Students

\section{Introduction}

Most of the cognitive abilities such as memory, perception and reasoning are achieved via the individuals' mental potentials. The sensory receptions and perceptions are complex processes which occur throughout the continuous trend of the individual's behavior. The sensory-motor activities are effective in identifying the body dimensions, its movement in the environment, and the spatial direction. It is indeed related to the place of the objects in the environment and the relation between that individual and the object (Coren, 2003). The main aim of the action or behavior in the sensory perception is the full recognition of the real environment that surrounds the human. Such recognition is claimed to prepare the human to become compatible with his environment, adjusting his behavior consistent with this recognition.

The external environment can be divided into the objects, events, ratios, and the relations among them. The most important ratios are the temporal and spatial ratios because we recognize the position and states of the objects by the ratio perception or the spatial relation while we understand the events' sequence by the perception of the temporal relation and ratio (shoarynezhad, 2010). The spatial ability is a mental ability originated from the structure of the human's intelligence. The intelligence is a general factor that includes a set of mental abilities which are divided into three groups, namely the spatial perception, spatial imagination, and mental rotation (Linn and Petersen, 1985).

The latter is the ability to visualize the rotation of one visual stimulus. The concept of the mental rotation was originally introduced by Shepard and Metzler (1971) .There are two notions about the mental rotation. While Cooper (1983) proclaims that in the mental rotation performance, the image is understood as a whole and the information obtained by 
the whole image is stored in the visual short-term memory, the counterpart notion accentuates that in the mental rotation, the propositional representations of the objects are given to the person and he performs the object rotation according to these data (Vandenberg and Kuse, 1978).

With regard to the structural and functional differences between the hemispheres of the brain, it is well agreed that in comparison with the left hemisphere, the right hemisphere is preferred in visual and spatial perceptions, as well as recognition of faces, objects, geometric shapes, and direction. Furthermore, the studies with Electroencephalogram have indicated that the electric activity undergoes an upsurge in the right hemisphere while performing a spatial task. The studies on the both hemispheres have also demonstrated that the right hemisphere is highly competent in the spatial sense and design recognition while it is preferred in reconstructing of geometric shapes over the left hemisphere. The mental rotation ability typically appears at the age four and before puberty (Subrahmanyam and Greenfield, 1994). In accordance with Vandenberg and Kuse's (1978) research, the mental rotation occurs via a constant rate while there is a relationship between the object's rotation angle and the speed of the mental rotation. This implies that the more the rotational angle of the object, the less will be the processing speed of the mental rotation. The spatial abilities play an imperative role in the initial stages of the perceptional activity learning and it is very crucial to employ them effectively. The mental rotation is the best part of the visual ability which serves the athletes' needs in majority of important conditions in sports through an appropriate and special motor response to some visual stimuli. Generally, the role of the visual system in the cognitive experience is evident while it is regarded as one of the visual performances related to information provided about the distant objects (assessment, color, and texture), the position, and speed (Guillot and Collet, 2005).

The cognitive skills such as prediction and decision making are really essential for the high level performances. These skills are effective with regard to the limited time and space in the sports and some strategic situations such as team players, fast and precise decision making in complex and unpredictable environments originated from various resources such as the ball, teammates, opponents, and the decision making process (Lim and Wang, 2009).

The reaction time has been recognized as one of the key parameters in most of the ball games which is necessary for most situations wherein the athletes are required to react to some visual stimuli with appropriate and special motor responses. One of the most paramount tasks for an athlete's mind during the game is to comprehend and incorporate the complex movement patterns. Additionally, this task is effective in the movements of the other players, the ball speed, unpredictable paths, and the abrupt changes in different directions or various obstructions (such as blocking the ball, the defender, or the player) (Faubert and Sidebottom, 2012).

The surveys have revealed that training the spatial skills is a principal factor in developing various movement skills as well as the problem solving ability. Such skills are indispensable for the individuals to perform their everyday tasks and complex skills in the body awareness and its various dimensions (Okagaki and Frensch, 1994).

Factors influential in the spatial ability have been claimed to be the age, sex, experience (its type and frequency), the structural difference, social factors, the cerebral asymmetry, and the talent. The researchers have established that when children are given training opportunities, they would react with more alacrity in response to the mental rotation.(Kail and Park, 1990).

According to the evolutionary-biological perspective of the brain, the brain growth and the intelligence in the girls is somewhat more than that of the boys during the childhood and adolescence periods (8-15 years old). Nonetheless, such a difference is not impressive. The fact is that the gender differences in the intelligence of children and adolescents are ignorable and unstable until the age 15. In line with this, many recommendations have been posed to describe the factors which contribute to gender differences in the mental rotation, among which hormonal, cultural, and motivational factors. Other contributing factors include the life before birth, sex hormones, and the impacts associated with the activation of testosterone levels (in the males 3 to 10 times more than the females). Another factor in boosting the boys' mental rotation ability is to inject testosterone as a male hormone (Parsons et al., 2004). In the western cultures and traditions, the spatial abilities are being predominantly considered as the males' conspicuous characteristics while boys are more than girls encouraged to participate in activities that increase their spatial skill developments (such as computer, mathematical, and scientific games). The mental rotation plays an important role in sports as well as the common routine errands that require more perception.

In the process of learning and improving the basketball skill, fast comprehension, the ability to have simultaneous retraining of the spatial relations, the speed of shape perception, and the ability to manipulate in the perceptual space as well as solving the problems in a short time are all important (Karalejić, Jakovljević, and Mandić, 2009). The karate is also a good illustration of a competitive sport which involves in high temporal levels and spatial restrictions requiring fast reactions in the combats and tournaments. The time reaction, speed, and the attack power are the most significant factors in assessing the karate techniques (Hooven, Chabris, Ellison, and Kosslyn, 2004). The specificity of hemispheres to spatial and verbal skills is more in the males than their counterparts. For the first time, Marmor (1975) performed the mental rotation in the children via a systematic method, demonstrating that even 5 years old kids used the mental rotation with 2-dimensional images for solving the problems. Yet, it was discerned that the 5 year old kids performed the mental rotation 2 times slower than the 8-year-olds. Linn and Petersen (1985) declared that after the age 13, boys could outperform in the mental rotation than the girl's peer group. Motor activity is one of the effective factors for increasing mental rotation. But in that wherein sport activities or in what sports, this skill increases, should be more 
studies done. With regard to the fact that mental rotation is one of important and effective factors in physical and educational activities and also this variable in men and women is different. So, with regard to these points, the main objective of this study is to compare the mental rotation performance in team and individual sports. Meanwhile, it is hypothesized that, there is a significance difference between these two groups.

\section{Methods}

The current research was a practical and causal-comparative study. The statistical population included all the female and male athletes $(\mathrm{N}=1500)$ from different districts of Shiraz, Iran who participated in the sport clubs under the supervision of the Education Department of Shiraz University, Iran. After all, 240 participants agreed to partake in our study. In details, 120 girls and 120 boys were selected by means of random sampling from four sport fields: Volleyball, Basketball, Karate, and Gymnastic. Indeed, 30 athletes were selected from each field randomly. Ethical approval for the study was obtained from the University of Shiraz, Faculty of Education and Psychology in Iran. The subjects were given a clear explanation of the objectives of the study. Finally, a consent forms was obtained for all subjects.

\subsection{Statistical society, sample and sampling method}

Mentrat Program, Software for the Mental Rotation Test was used in this study. This mental rotation Software was assessed via the Psychlab website. This website was originally developed by Chi, Pirolli, and Pitkow (2000) and is based on Vandenberg and Kuse (1978) Mental Rotations Test(MRT). This test included 32 items and 2 blocks of 16 trails and each trail was presented by "F" on the computer screen.

This figure had 8 rotations and it rotated in the different angles (vertical, $45^{\circ}, 90^{\circ}, 135^{\circ}, 180^{\circ}, 225^{\circ}, 270^{\circ}, 315^{\circ}$ ) while the participant were required to recognize whether the presented image was rotating or mirroring. The participants responded via two keys on the keyboard. Finally, the software calculated the number of right and wrong responses in addition to computing the test time in each period. In this study, obtained Cronbach's alpha was 0.62 .

\subsection{Instruments}

First, one Demographic Questionnaire, encompassing various questions about the age, sex, history, and the sport field, was distributed among the participants. The mental rotation test was performed by using the mental rotation test software from the Psychlab website. It needs to be highlighted these instruments were also used in studies such as Rafi, Samsudin, and Ismail (2006), Samsudin and Ismail (2004). The test included 2 blocks of 16 trails and each trail was presented by "F" in 8 rotations from different angles. The participants were asked to recognize whether the presented image was rotating or mirroring. The participants responded via two keys on the keyboard. The test lasted for 3 to 4 minutes. The data were analyzed by SPSS software Version 19 (SPSS, Inc., Chicago, IL, USA). In order to compare the groups, the Analysis of Variance with repeated measures was applied at a significance level of $\alpha=0.05$.

\section{Results}

Table 1 presents the means and standard deviations for both male and female groups in different sport fields. The highest and the lowest means of age (13.5 and 12.6) respectively belonged to the boys in the Volleyball field and the girls in the Gymnastics filed. Concerning the sport history, the highest mean and the lowest mean belonged to the boys in the Gymnastics field (3.2) and the girls in the Basketball filed (1.93), respectively.

Table1. The participants' descriptive statistics o in both male and female group in different sport fields

\begin{tabular}{ccccc}
\hline Sport Fields & Sex & Number & Age(years) & Sport History (years) \\
\hline Volleyball & female & 30 & $13.3 \pm 1.3$ & $2.0 \pm 1.5$ \\
\cline { 2 - 5 } & male & 30 & $13.5 \pm 1.2$ & $2.8 \pm 1.4$ \\
\hline Basketball & female & 30 & $13.1 \pm 1.2$ & $1.9 \pm 1.3$ \\
\cline { 2 - 5 } & male & 30 & $12.9 \pm 1.1$ & $2.8 \pm 1.8$ \\
\hline Karate & female & 30 & $12.9 \pm 1.3$ & $2.3 \pm 1.6$ \\
\cline { 2 - 5 } & male & 30 & $13.0 \pm 1.7$ & $2.3 \pm 1.5$ \\
\hline Gymnastics & female & 30 & $12.6 \pm 1.0$ & $2.8 \pm 1.8$ \\
\cline { 2 - 5 } & male & 30 & $13.2 \pm 1.1$ & $3.2 \pm 1.8$ \\
\hline
\end{tabular}

The ANOVA results with the repeated measures for the boys and girls of the team sports are shown in Table 2 , proving that all the impacts were significant between the rotational angles of the participants in the different rotations $(\mathrm{F}=21.03, \mathrm{P}=.000)$. It means that as the rotational angle of the object increases, the number of correct responses will be decreased. Nonetheless, the effects of the group and sex (the boy and the girl in the team) $(\mathrm{F}=0.03)$ as well as the interaction of the rotation angle and the group were not significant $(\mathrm{F}=1.73, \mathrm{P}=0.09)$. 
Table 2. The ANOVA results with the repeated measures for the boys and girls of the team sports

\begin{tabular}{lccccc}
\hline Source & $\begin{array}{c}\text { Sum of } \\
\text { squares }\end{array}$ & df & mean & F & $p$ \\
\hline Rotational Angle & 128.45 & 7 & 18.35 & 21.03 & $0.000^{*}$ \\
\hline Group & 0.15 & 1 & 0.15 & 0.03 & 0.84 \\
\hline $\begin{array}{l}\text { Rotational Angle } \times \\
\text { Group }\end{array}$ & 10.56 & 7 & 1.51 & 1.73 & 0.09 \\
\hline
\end{tabular}

$* \mathrm{p}<0.05$

As it is observed in Table 3, the impact of the rotation angle $\mathrm{F}=7.27$ and $\mathrm{P}=0.000$ was significant but the impacts of the group $(\mathrm{F}=0.67, \mathrm{P}=0.41)$ and the interaction of the rotation angle with the group $(\mathrm{F}=0.80, \mathrm{P}=0.58)$ were insignificant.

Table 3. The ANOVA results with the repeated measures for the boys and girls of the individual sports

\begin{tabular}{lccccc}
\hline Source & Sum of squares & $\mathrm{df}$ & mean & $\mathrm{F}$ & $\mathrm{p}$ \\
\hline Rotational Angle & & & & & \\
\hline Group & 31.56 & 7 & 4.50 & 7.27 & 0.000 \\
\hline Rotational Angle $\times$ Group & 2.8 & 1 & 2.8 & 0.67 & 0.41 \\
\hline$* \mathrm{p}<0.05$ & 3.46 & 7 & 0.49 & 0.80 & 0.58 \\
\hline
\end{tabular}

Table 4 displays that the impact of the rotation angle $F=13.63$, the impact of the group $F=4.5$ and their interactions $F=4.49$ were significant. Significance of the group impact means that there was a significant difference between the mean of the correct responses of the boys in the team and individual groups. Significance of the interaction also implies that both groups were different from various perspectives (with regard to the type of sport fields).

Table 4. ANOVA results with the repeated measures for the boys of both individual and team sports

\begin{tabular}{lccccc}
\hline Source & $\begin{array}{c}\text { Sum of } \\
\text { squares }\end{array}$ & df & mean & F & p \\
\hline Rotational Angle & 74.6 & 7 & 10.58 & 13.63 & 0.000 \\
\hline Group & 18.5 & 1 & 18.5 & 4.50 & 0.03 \\
\hline Rotational Angle $\times$ Group & 24.42 & 7 & 3.49 & 4.49 & 0.000 \\
\hline
\end{tabular}

$* \mathrm{p}<0.05$

\section{Discussion and conclusion}

The purpose of the present study was to compare the mental rotation performance in team and individual sports of the students in Shiraz. The rotation angle was significant in both groups $(p=0.000)$. As the shape of letter " $F$ " was changed from the vertical status (on the basis of different degrees) and the length of the response time with the test decision making increases, it can be concluded that in the decision making process, the participants primarily rotate the presented image in their minds to obtain a vertical status and then they probe it and make their judgments.

In another study by Pietsch and Jansen (2012), the impact of the mental rotation was assessed in three groups of students majoring in Music, Sport Sciences, and the Educational Sciences. The results proved that the students of music and sport sciences had a better mental rotation than the ones studying educational sciences. Also the studies by Rilea, Roskos-Ewoldsen, and Boles (2004) indicated that the type of task stimulus exerted an influence on the processing task. Likewise, Kass, Ahlers, and Dugger (1998) showed that using some computer games and tasks were more effective in the mental rotation of girls than that of the boys. Jansen and Lehmann (2013) addressed the mental rotation performance in Soccer player, Gymnasts, and nonathletic people, finding that the gymnasts outshined the others in the mental rotation. Most of them were trained to rotate around three axes. Another study was conducted by Jansen, Lehmann, and Van Doren (2012) to deal with the mental rotation of male soccer players and nonathletic people to determine their spatial-visual abilities. It was found out that the soccer players had faster reaction compared to the nonathletic individuals. Because different impacts of the spatial ability, especially the mental rotation, on the physical activity is 
thoroughly accepted, the present study was designed to compare the mental rotation in two groups of team and individual sports among both girls and boys.

It needs to be mentioned that the results of this study are consistent with the results reported by (Shepard and Metzler, 1971; Vandenberg and Kuse, 1978; Jansen, Schmelter, Kasten, and Heil, 2011; Quaiser-Pohl, Geiser, and Lehmann, 2006; Roberts and Bell, 2000), and while no result could be found in contradiction to the results of our study. At this juncture, it is worth noting that the types of sport fields were different in each previously-conducted study while leaving us with no research about the various fields.

The results of this study demonstrated that the mental rotation performance in two groups of boys and girls in team sports (such as Volleyball and Basketball) was not significant. Bearing in mind the proven relation between the mental rotation and the movement process, , it was observed that there was no significant difference between the performances of the mental rotation in the two groups of boys and girls (team sports) and both groups had a similar performance. Yet, as it was mentioned earlier, the physical activity is one of the most effective factors that increase the performance of the mental rotation. The Neurology description also indicates that there is anatomic difference in some areas that play important roles in the movement processing. Meanwhile, the positive effects of the spatial abilities have been proved in the sports.

Comparing the mental rotation performance in two groups of girls and boys in the individual sports (such as Karate and Gymnastic), it was approved that the mental rotation was not significant in these groups. Consequently, no study about the comparison of mental rotation performance was found in the individual sports. Due to the fact that recent studies have shown the decrease in the gap between boys and girls in terms of their spatial abilities and that both groups have physical activities, there were fewer individual differences in our study while both groups exhibited similar performances in individual sports. In addition, gender differences in the mental rotation ability can be discussed from the biological point of view. As for the sex, the results of this study were not in compliance with the ones reported by (Vandenberg and Kuse, 1978; Quaiser-Pohl et al, 2006; Parsons et al, 2004; and Voyer et al, 1995).

According to the Evolutional-Biological view, the brain development and intelligent rates in the girls are somewhat more than the boys during the childhood (8-15 years old) but this difference is not significant. The impact of gender differences on the intelligence of children and adolescents is slight and unstable until the age 15 but after 16 , both the brain size and the intelligent rate in boys will be more than their counterparts, peaking in the adulthood and remaining stable henceforth (Lynn, 1999). Among most cultures, the gender role pattern is similar in the visual -spatial ability, advocating the principles of the evolutionary psychology. Such intercultural data shows that although for the gender differences, some general patterns have been recognized worldwide, the gender role in the cognition ability is different over various cultures.

\section{References}

Chi, E. H., Pirolli, P., and Pitkow, J. (2000). The scent of a site: A system for analyzing and predicting information scent, usage, and usability of a web site. In Proceedings of the SIGCHI conference on Human Factors in Computing Systems (pp. 161-168). ACM.

Cooper, L. A. a. R., D.T. (1983). Attention,Perception and intelligence. NewYork:Cambridge University Press.

Coren, S. (2003). Sensation and perception: Wiley Online Library.

Faubert, J., and Sidebottom, L. (2012). Perceptual-cognitive training of athletes. Journal of Clinical Sport Psychology, $6(1), 85$.

Guillot, A., and Collet, C. (2005). Duration of mentally simulated movement: a review. Journal of motor behavior, $37(1), 10-20$.

Hooven, C. K., Chabris, C. F., Ellison, P. T., and Kosslyn, S. M. (2004). The relationship of male testosterone to components of mental rotation. Neuropsychologia, 42(6), 782-790.

Jansen, P., and Lehmann, J. (2013). Mental rotation performance in soccer players and gymnasts in an object-based mental rotation task. Advances in Cognitive Psychology, 9(2), 92.

Jansen, P., Lehmann, J., and Van Doren, J. (2012). Mental rotation performance in male soccer players. PloS one, 7(10), e48620.

Jansen, P., Schmelter, A., Kasten, L., and Heil, M. (2011). Impaired mental rotation performance in overweight children. Appetite, 56(3), 766-769.

Johnson, A. M. (1990). Speed of mental rotation as a function of problem-solving strategies. Perceptual and motor skills, 71(3), 803-806.

Kail,R.,andPark,Y (1990). Impact of practice on speed of mental rotation. Journal of Experimental Child Psychology, 49(2), 227-244

Karalejić, M., Jakovljević, S., and Mandić, R. (2009). Relations between basketball skills and certain cognitive abilities of junior basketball players. Fizička kultura, 63(1), 60-75. 
Kass, S. J., Ahlers, R. H., and Dugger, M. (1998). Eliminating gender differences through practice in an applied visual spatial task. Human Performance, 11(4), 337-349.

Lim, B., and Wang, C. (2009). Perceived autonomy support, behavioural regulations in physical education and physical activity intention. Psychology of Sport and Exercise, 10(1), 52-60.

Linn, M. C., and Petersen, A. C. (1985). Emergence and characterization of sex differences in spatial ability: A metaanalysis. Child development, 1479-1498.

Lynn, R. (1999). Sex differences in intelligence and brain size: A developmental theory. Intelligence, 27(1), 1-12.

Marmor, G. S. (1975). Development of kinetic images: When does the child first represent movement in mental images? Cognitive Psychology, 7(4), 548-559.

Okagaki, L., and Frensch, P. A. (1994). Effects of video game playing on measures of spatial performance: Gender effects in late adolescence. Journal of applied developmental psychology, 15(1), 33-58.

Parsons, T. D., Larson, P., Kratz, K., Thiebaux, M., Bluestein, B., Buckwalter, J. G., and Rizzo, A. A. (2004). Sex differences in mental rotation and spatial rotation in a virtual environment. Neuropsychologia, 42(4), 555-562.

Pietsch, S., and Jansen, P. (2012). Different mental rotation performance in students of music, sport and education. Learning and Individual Differences, 22(1), 159-163.

Quaiser-Pohl, C., Geiser, C., and Lehmann, W. (2006). The relationship between computer-game preference, gender, and mental-rotation ability. Personality and Individual Differences, 40(3), 609-619.

Rafi, A., Samsudin, K. A., and Ismail, A. (2006). On improving spatial ability through computer-mediated engineering drawing instruction. Journal of educational technologyand society, 9(3), 149.

Rilea, S. L., Roskos-Ewoldsen, B., and Boles, D. (2004). Sex differences in spatial ability: A lateralization of function approach. Brain and cognition, 56(3), 332-343.

Roberts, J. E., and Bell, M. A. (2000). Sex differences on a mental rotation task: variations in electroencephalogram hemispheric activation between children and college students. Developmental neuropsychology, 17(2), 199-223.

Samsudin, K. A., and Ismail, A. (2004). The improvement of mental rotation through computer based multimedia tutor. Malaysian Online Journal of Instructional Technology (MOJIT), 1(2), 24-34.

Shepard, R. N., and Metzler, J. (1971). Mental rotation of three-dimensional objects. Science, 972, 701-703.

shoarynezhad. (2010). Developmental Psychology (19 ed.). Tehran: dissemination of information.

Subrahmanyam, K., and Greenfield, P. M. (1994). Effect of video game practice on spatial skills in girls and boys. Journal of applied developmental psychology, 15(1), 13-32.

Vandenberg, S. G., and Kuse, A. R. (1978). Mental rotations, a group test of three-dimensional spatial visualization. Perceptual and motor skills, 47(2), 599-604.

Voyer, D., Voyer, S., and Bryden, M. P. (1995). Magnitude of sex differences in spatial abilities: a meta-analysis and consideration of critical variables. Psychological bulletin, 117(2), 250. 